\title{
Electroweak vector boson production at the LHC as a probe of mechanisms of diffraction
}

\author{
Krzysztof Golec-Biernat, ${ }^{1,2}$ Christophe Royon, ${ }^{3}$ Laurent Schoeffel, ${ }^{3}$ and Rafal Staszewski ${ }^{1}$ \\ ${ }^{1}$ Institute of Nuclear Physics Polish Academy of Sciences, Kraków, Poland \\ ${ }^{2}$ Institute of Physics, University of Rzeszów, Rzeszów, Poland \\ ${ }^{3}$ CEA Saclay, Irfu/SPP, 91191 Gif/Yvette Cedex, France
}

\begin{abstract}
We show that the double diffractive electroweak vector boson production in the $p p$ collisions at the LHC is an ideal probe of QCD based mechanisms of diffraction. Assuming the resolved Pomeron model with flavor symmetric parton distributions, the $W$ production asymmetry in rapidity equals exactly zero. In other approaches, like the soft color interaction model, in which soft gluon exchanges are responsible for diffraction, the asymmetry is non-zero and equal to that in the inclusive $W$ production. In the same way, the ratio of the $W$ to $Z$ boson production is independent of rapidity in the models with resolved Pomeron in contrast to the predictions of the soft color interaction model.
\end{abstract}

Keywords: diffractive processes, electroweak bosons, quantum chromodynamics

\section{INTRODUCTION}

Measurements of the charge asymmetry of leptons originating from the decay of singly produced $W^{ \pm}$bosons at $p p$, $p \bar{p}$ and $e p$ colliders provides important information about the proton structure as described by parton distribution functions (PDFs) [1 [6]. In particular, a direct access to these distributions is provided by the measurement of the $W^{ \pm}$bosons production asymmetry in rapidity. This quantity reflects the fact that at given rapidity the two charged vector bosons are produced by quarks of different flavors.

In the double diffractive exchange, the two colliding hadrons remain intact creating two gaps in rapidity where no hadronic activity is expected. Consequently, the production of electroweak vector bosons in such processes is done in association with the two gaps in rapidity. The production of electroweak vector bosons is a QCD process with a hard scale, given by the boson mass, thus it can be described perturbatively. However, the QCD based understanding of the rapidity gap formation remains a challenge [7 9]. We should mention at this point that in the measurements of diffractive events at the LHC the key element is to tag the forward scattered incoming protons. Because of the pile up of events in each bunch crossing (at high luminosities up to 35 pile up events per bunch crossing occur), the rapidity gap tagging is no longer possible, and the only possibility to detect double diffractive events is by tagging the intact protons in the final state.

In the resolved Pomeron model interpretation [10], the scattered protons stay intact and rapidity gaps are created due to exchange of two Pomerons with a partonic structure. Thus, the electroweak bosons are diffractively produced from the annihilation of two quarks coming from each of the two Pomerons. In other models, like the soft color interaction model [11], the diffractive gaps are produced due to soft gluon exchanges which neutralize color in the rapidity space between two outgoing protons and the diffractive system. In this type of models, the hard process is independent of the gap formation mechanisms and is the same as in the non-diffractive events 7,8$]$.

In order to discriminate between these two essentially different mechanisms of diffraction 10, 11] we propose to study the $W^{ \pm}$rapidity asymmetry for double diffractive exchanges. In the Pomeron exchange models, the quark content of the Pomeron is flavor symmetric in order to account for the vacuum quantum number exchange between the scattered protons and the diffractive system. Therefore, the $W$ production asymmetry is exactly equal to zero. This stays in the contrast to the result from the soft color interaction models in which the $W$ asymmetry is the same as in the inclusive case, determined by the $u$ and $d$ quark content of the proton. In the same spirit, the $W$ to $Z$ production rate can be studied, showing independence of rapidity in the Pomeron models and being given by the shape from the inclusive case in the soft color interaction model. Thus, we reach the conclusion that the study of the double diffractive electroweak boson production at the LHC is an ideal test of the two distinct QCD mechanisms responsible for diffractive processes in hadronic collisions. 
$\mathrm{W}$ and $\mathrm{Z}$ production cross sections at LHC



W asymmetry at LHC

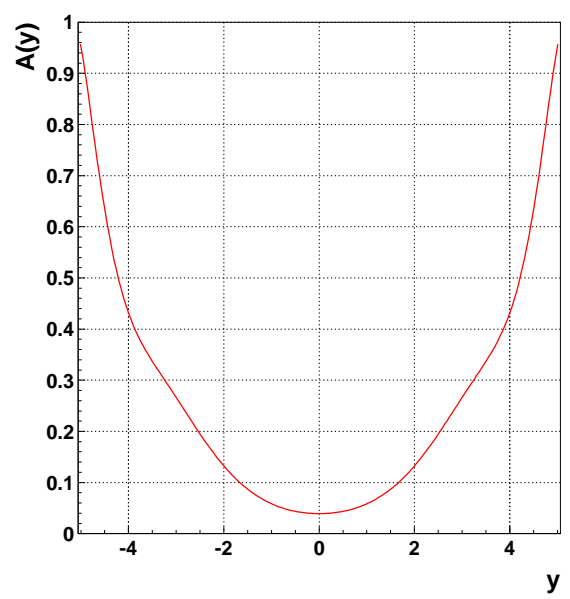

FIG. 1: The $W$ and $Z$ boson production cross sections at the LHC as a function of the boson rapidity $y$ (left) and the corresponding $W$ boson rapidity asymmetry (right) for the LO MSTW08 parton distributions.

\section{II. $W$ BOSON RAPIDITY ASYMMETRY}

\section{A. Non-diffractive case}

The electroweak vector bosons are produced in the $p p$ scattering from annihilation of two quarks. The boson rapidity $y$ is determined by the longitudinal proton momentum fractions, $x_{1}$ and $x_{2}$, carried by the colliding quarks

$$
x_{1}=\frac{M_{W}}{\sqrt{s}} \mathrm{e}^{y}, \quad x_{2}=\frac{M_{W}}{\sqrt{s}} \mathrm{e}^{-y}
$$

where $M_{W}$ is the $W$ boson mass and $\sqrt{s}$ is invariant energy of scattering protons. From the conditions $0 \leq x_{1,2} \leq 1$, we find the following bound for the $W$ rapidity

$$
-y_{\min } \leq y \leq y_{\min }
$$

where $y_{\min }=\ln \left(\sqrt{s} / M_{W}\right)$. The measured inclusively $W^{ \pm}$rapidity asymmetry,

$$
A_{\text {incl }}(y)=\frac{d \sigma_{W^{+}}(y) / d y-d \sigma_{W^{-}}(y) / d y}{d \sigma_{W^{+}}(y) / d y+d \sigma_{W^{-}}(y) / d y},
$$

is determined mainly by the $u_{p}$ and $d_{p}$ quark distributions in the colliding protons. With a simplifying assumption that the anti-quark distributions in the proton are equal, $\bar{u}=\bar{d}$, we have [12]

$$
A_{\text {incl }}(y)=\frac{\left(u_{p}\left(x_{1}\right)-d_{p}\left(x_{1}\right)\right) \bar{u}_{p}\left(x_{2}\right)+\bar{u}_{p}\left(x_{1}\right)\left(u_{p}\left(x_{2}\right)-d_{p}\left(x_{2}\right)\right)}{\left(u_{p}\left(x_{1}\right)+d_{p}\left(x_{1}\right)\right) \bar{u}_{p}\left(x_{2}\right)+\bar{u}_{p}\left(x_{1}\right)\left(u_{p}\left(x_{2}\right)+d_{p}\left(x_{2}\right)\right)}
$$

where the quark distributions also depend on a hard scale given by the $W$ boson mass $\mu=M_{W}$. The measured non-zero value of $A_{\text {incl }}(y)$ is explained by different distributions of $u$ and $d$ quarks in the proton: $u_{p} \neq d_{p}$. Thus, the $W$ rapidity asymmetry is a good observable to pin down these distributions and is usually included in global fits of parton distribution functions in a nucleon. The $W$ and $Z$ boson production cross sections and the corresponding $W$ rapidity asymmetry are shown in Fig. 1]in the non-diffractive case for the proton (with the strange quark contribution included), where the leading order MSTW08 parametrization of PFDs was used [13].

\section{B. Diffractive case}

In the double diffractive production, the electroweak bosons are parts of a diffractive system with mass $M_{D}$ which is separated in rapidity from the scattered protons by two gaps of the size $\ln \left(1 / \xi_{1}\right)$ and $\ln \left(1 / \xi_{2}\right)$, where $\xi_{1,2}$ are small 
fractions of the incoming proton momenta transferred into the diffractive system. They obey the following relation

$$
\xi_{1} \xi_{2}=\frac{M_{D}^{2}}{s}, \quad \xi_{1,2} \ll 1 .
$$

Thus, rapidity of the diffractively produced $W$ boson stays in the range

$$
-y_{\min }+\ln \left(1 / \xi_{1}\right) \leq y \leq y_{\min }-\ln \left(1 / \xi_{2}\right)
$$

The relevant $W$ rapidity asymmetry is defined now as the ratio of triple differential cross sections

$$
A_{D}\left(y, \xi_{1}, \xi_{2}\right)=\left(\frac{d \sigma_{W^{+}}}{d y d \xi_{1} d \xi_{2}}-\frac{d \sigma_{W^{-}}}{d y d \xi_{1} d \xi_{2}}\right) /\left(\frac{d \sigma_{W^{+}}}{d y d \xi_{1} d \xi_{2}}+\frac{d \sigma_{W^{-}}}{d y d \xi_{1} d \xi_{2}}\right)
$$

\section{QCD INTERPRETATIONS OF DIFFRACTIVE $W$ BOSON RAPIDITY ASYMMETRY}

The electroweak boson mass is a hard scale which allows for perturbative QCD interpretation of the $W$ or $Z$ production. However, in the diffractive production of electroweak vector bosons there are several approaches to the nature of the vacuum quantum number exchange which leads to rapidity gaps.

In the resolved Pomeron exchange interpretation, the Pomeron is endowed with a partonic structure described by the Pomeron parton distributions. The double diffractive processes can then be qualified as a double Pomeron exchange (DPE) with vacuum quantum numbers. In this case, the ordinary parton distributions in the proton are replaced by diffractive parton distribution functions (DPDFs), $q_{D}(x, \xi)$ for quarks and $g_{D}(x, \xi)$ for gluons. They encode the information about momentum fractions $\xi$ transferred from the initial proton into the diffractive system [10, 14 17]. An additional assumption is usually made that the diffractive parton distributions have a factorized form

$$
q_{D}(x, \xi, \mu)=\Phi(\xi) q_{\mathbb{P}}(\beta, \mu),
$$

where $\beta=x / \xi$ is a fraction of the Pomeron momentum carried by a quark participating in the diffractive scattering. We also indicated the hard scale dependence of these distributions, $\mu=M_{W}$ in our case. The quantity $q_{\mathbb{P}}(\beta, \mu)$ is called a Pomeron quark distribution, and $\Phi(\xi)$ represents a Pomeron flux. In this way, the vacuum exchange aspect of diffractive processes separated from the hard scattering of two quarks which produces the $W$ boson. However, the assumption about the factorization (8) is not necessary in our considerations.

The leading order cross sections for the DPE models of the $W$ boson production are given by the inclusive case formula [12] with the diffractive quark distributions

$$
\begin{aligned}
& \frac{d^{3} \sigma_{W^{+}}}{d y d \zeta_{1} d \zeta_{2}}=\sigma_{0}^{W} V_{u d}^{2}\left\{u_{D}\left(x_{1}, \xi_{1}\right) \bar{d}_{D}\left(x_{2}, \xi_{2}\right)+\bar{d}_{D}\left(x_{1}, \xi_{1}\right) u_{D}\left(x_{2}, \xi_{2}\right)\right\} S^{2} \\
& \frac{d^{3} \sigma_{W^{-}}}{d y d \zeta_{1} d \zeta_{2}}=\sigma_{0}^{W} V_{u d}^{2}\left\{d_{D}\left(x_{1}, \xi_{1}\right) \bar{u}_{D}\left(x_{2}, \xi_{2}\right)+\bar{u}_{D}\left(x_{1}, \xi_{1}\right) d_{D}\left(x_{2}, \xi_{2}\right)\right\} S^{2}
\end{aligned}
$$

where $\sigma_{0}^{W}=2 \pi G_{F} M_{W}^{2} /(3 \sqrt{2} s)$ and $V_{u d}$ is the CKM matrix element. In addition, we multiply the cross sections by a survival probability [18] of the DPE process, $S^{2}$, which might be a complicated function of the fractions $\xi_{1,2}$. We also neglected the Cabbibo suppressed $s$ quark part of the $W$ boson production cross sections [12] . Note that adding NLO and NNLO corrections to cross section expressions above [6] is not important for the idea developed in this paper on asymmetries.

The $W$ boson asymmetry (7) computed from the above cross sections is given by formula (4) with the ordinary quark distributions replaced by the diffractive ones. Since the Pomeron carries vacuum quantum numbers, it is expected to be made of gluons and sea quarks. Then, diffractive PDFs in eqs. (9) and (10) have to follow the relations: $u_{D}=\bar{u}_{D}$ and $d_{D}=\bar{d}_{D}$. Thus, the $W^{ \pm}$boson production asymmetry in the DPE models equals zero,

$$
A_{D}\left(y, \xi_{1}, \xi_{2}\right)=0
$$

independent of the rapidity gap sizes determined by the fractions $\xi_{1,2}$.

The $W^{ \pm}$and $Z$ cross sections are displayed in Fig. 2 (top) for the pomeron-based models with and without taking into account the ATLAS Forward Proton (AFP) detectors [19, 20]. The AFP project consists in installing forward proton detectors located at $220 \mathrm{~m}$ and $420 \mathrm{~m}$ from the ATLAS interaction point. In the first phase of the project, only the detectors at $220 \mathrm{~m}$ are considered which leads to an acceptance in $\xi$, the proton momentum loss, of $0.01<\xi<0.15$ and this acceptance is assumed in the following of the paper. Let us note that the acceptance increases down to 0.002 



FIG. 2: Top: diffractive $W$ and $Z$ boson production cross sections as a function of rapidity in the DPE model with and without taking into account the AFP acceptance (see the text). Bottom: the same cross sections in the SCI model.

if detectors at $420 \mathrm{~m}$ are added, which is fundamental to detect the exclusive production of lower mass objects, such as the Higgs bosons [21, 22]. In Fig. 2 is displayed the number of $Z$ and $W$ events observed in the AFP acceptance for a luminosity of $10 \mathrm{fb}^{-1}$, which is quite low at the LHC [20]. We note the high number of events measured even at a relatively low luminosity which allows to probe quantitatively the different models of diffraction, as we will see in the following.

The soft color interaction approach (SCI) modifies the color reconnection at the hadronization level in order to produce rapidity gaps. Then, the diffractive PDFs are not needed and up to correction factors originating from 


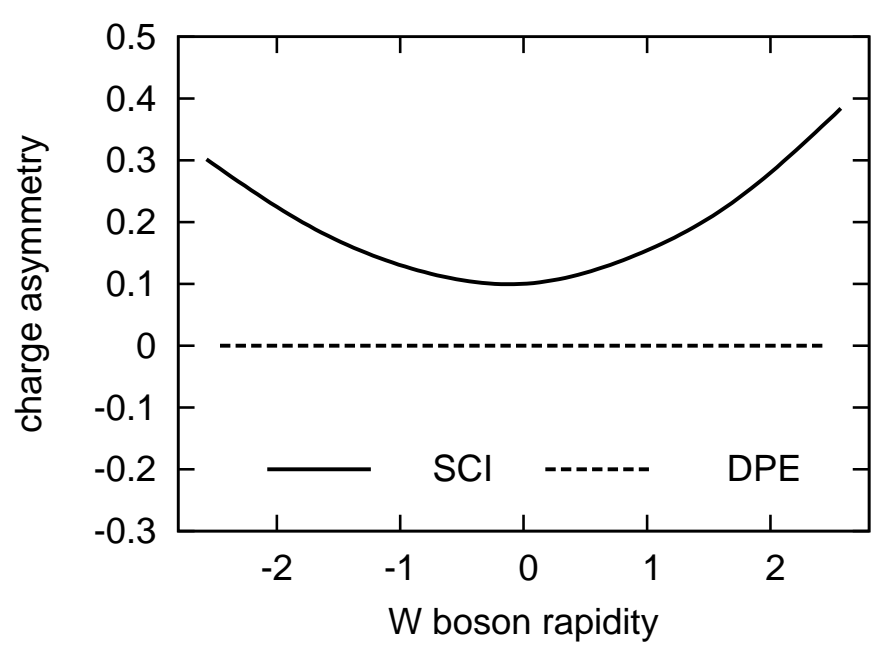

FIG. 3: $W$ asymmetries as a function of the $W$ boson rapidity $y$ in the pomeron based (DPE) and soft color interaction (SCI) models, using the AFP acceptance. We note the flat distribution for the DPE models and a strong $y$ dependence for the SCI models.

features of the hadronization process, the $W$ boson production cross sections read as for the standard inclusive case

$$
\begin{aligned}
& \frac{d^{3} \sigma_{W^{+}}}{d y d \zeta_{1} d \zeta_{2}} \propto \sigma_{0}^{W} V_{u d}^{2}\left\{u_{p}\left(x_{1}\right) \bar{d}_{p}\left(x_{2}\right)+\bar{d}_{p}\left(x_{1}\right) u_{p}\left(x_{2}\right)\right\} \\
& \frac{d^{3} \sigma_{W^{-}}}{d y d \zeta_{1} d \zeta_{2}} \propto \sigma_{0}^{W} V_{u d}^{2}\left\{d_{p}\left(x_{1}\right) \bar{u}_{p}\left(x_{2}\right)+\bar{u}_{p}\left(x_{1}\right) d_{p}\left(x_{2}\right)\right\}
\end{aligned}
$$

with the same proportionality coefficient. Thus, we expect the asymmetry (7) to be equal or be very close to the non-zero value given by the standard $W$ rapidity asymmetry (4),

$$
A_{D}\left(y, \xi_{1}, \xi_{2}\right)=A_{\text {incl }}(y) \text {, }
$$

independent of the rapidity gap sizes. The $W^{ \pm}$cross sections in the SCI approach are shown in Fig. 2 (bottom). They have have to be multiplied by a rapidity gap survival factor [18]. Note that the global factors in the SCI model that multiply the above expressions disappear in the asymmetry ratio. In this way, by measuring the $W$ rapidity asymmetry in the double diffractive processes, we are able to discriminate between the DPE and SCI mechanisms of diffraction in the $p p$ scattering.

We summarize this conclusion in Fig. 3 where the $W$ asymmetries are shown in the case of the pomeron and the soft color interaction based models. The $y$-dependence of the $W$ asymmetry is expected to be flat and close to zero in the DPE models whereas for the SCI models it is close to the non-diffractive $W$ asymmetry in the $p p$ scattering case. Distinguishing between both models will be easy at the LHC, given the cross section of those processes and the luminosity available.

\section{IV. $W / Z$ PRODUCTION RATIO: DISTINCTION BETWEEN DPE AND SCI MODELS}

We also consider the $Z$ production rate, determined in the inclusive case by the leading order cross section

$$
\frac{d \sigma_{Z}}{d y}=\sigma_{0}^{Z}\left\{C_{u} u_{p}\left(x_{1}^{\prime}\right) \bar{u}_{p}\left(x_{2}^{\prime}\right)+C_{d} d_{p}\left(x_{1}^{\prime}\right) \bar{d}_{p}\left(x_{2}^{\prime}\right)+\left(x_{1}^{\prime} \leftrightarrow x_{2}^{\prime}\right)\right\}
$$

with $\sigma_{0}^{Z}=2 \pi G_{F} M_{Z}^{2} /(3 \sqrt{2} s), C_{u, d}=V_{u, d}^{2}+A_{u, d}^{2}$ where $V_{u, d}=T_{u, d}^{3}-2 Q_{u, d} \sin ^{2} \theta_{W}$ and $A_{u, d}=T_{u, d}^{3}$ are the vector and axial couplings of $u$ and $d$ quarks to $Z$ boson. Notice that the PDFs are computed at the factorization scale $\mu=M_{Z}$, and the momentum fractions are also related to $Z$ boson mass, $x_{1}^{\prime}=M_{Z} \mathrm{e}^{ \pm y} / \sqrt{s}$. The $W$ to $Z$ production ratio,

$$
R_{\text {inc }}(y) \equiv \frac{1}{2}\left(\frac{d \sigma_{W}^{+}}{d y}+\frac{d \sigma_{W}^{+}}{d y}\right) / \frac{d \sigma_{Z}}{d y}
$$


in the inclusive case reads

$$
R_{\mathrm{incl}}(y)=\frac{M_{W}^{2} V_{u d}^{2}}{M_{Z}^{2}} \frac{u_{p}\left(x_{1}\right) \bar{d}_{p}\left(x_{2}\right)+\bar{d}_{p}\left(x_{1}\right) u_{p}\left(x_{2}\right)+d_{p}\left(x_{1}\right) \bar{u}_{p}\left(x_{2}\right)+\bar{u}_{p}\left(x_{1}\right) d_{p}\left(x_{2}\right)}{C_{u} u_{p}\left(x_{1}^{\prime}\right) \bar{u}_{p}\left(x_{2}^{\prime}\right)+C_{d} d_{p}\left(x_{1}^{\prime}\right) \bar{d}_{p}\left(x_{2}^{\prime}\right)+\left(x_{1}^{\prime} \leftrightarrow x_{2}^{\prime}\right)} .
$$

The distributions in the numerator are taken at the scale $\mu=M_{W}$ (and $x_{1,2}=M_{W} \mathrm{e}^{ \pm y} / \sqrt{s}$ ) while for those in the denominator $\mu=M_{Z}$.

In the DPE model, in eq. (15) the ordinary proton PDFs are replaced by the diffractive PDFs and additionally, the cross section is multiplied by a survival factor $S^{2}$,

$$
\frac{d^{3} \sigma_{Z}}{d y d \xi_{1} d \xi_{2}}=\sigma_{0}^{Z}\left\{C_{u} u_{D}\left(x_{1}^{\prime}, \xi_{1}\right) \bar{u}_{D}\left(x_{2}^{\prime}, \xi_{2}\right)+C_{d} d_{D}\left(x_{1}^{\prime}, \xi_{1}\right) \bar{d}_{D}\left(x_{2}^{\prime}, \xi_{2}\right)+\left(\left(x_{1}^{\prime}, \xi_{1}\right) \leftrightarrow\left(x_{2}^{\prime}, \xi_{2}\right)\right)\right\} S^{2} .
$$

The corresponding $W$ to $Z$ production ratio, defined by the formula

$$
R_{D}\left(y, \xi_{2}, \xi_{2}\right) \equiv \frac{1}{2}\left(\frac{d^{3} \sigma_{W}^{+}}{d y d \xi_{1} d \xi_{2}}+\frac{d^{3} \sigma_{W}^{-}}{d y d \xi_{1} d \xi_{2}}\right) / \frac{d^{3} \sigma_{Z}}{d y d \xi_{1} d \xi_{2}}
$$

has the form (17) with the diffractive PDFs. The multiplicative survival factor $S^{2}$ cancels out in this ratio. The ratio $R_{D}$ for the DPE model is shown as the dashed line in Fig. 4. Assuming the flavour symmetry of the diffractive PDFs, $u_{D}=\bar{u}_{D}=d_{D}=\bar{d}_{D}$, we find an almost constant ratio in the central rapidity region

$$
R_{D}\left(y, \xi_{2}, \xi_{2}\right) \approx \frac{M_{W}^{2} V_{u d}^{2}}{M_{Z}^{2}} \frac{1}{C_{u}+C_{d}}
$$

A much larger deviation from a constant value occurs close to the rapidity gap edges, see Fig. 4

In the SCI model, however, the ratio depends on rapidity through the ordinary quark distributions in the proton, and we obtain

$$
R_{D}\left(y, \xi_{2}, \xi_{2}\right)=R_{\text {incl }}(y) .
$$

This ratio is shown in Fig. 4 as the solid line. We notice again that the $W$ to $Z$ cross section ratio is an additional observable allowing to distinguish between the DPE and SCI models.

\section{TEST OF FLAVOR SYMMETRY OF DIFFRACTIVE PDFS}

The almost constant ratio $R_{D}$ in the DPE models was obtained under the assumption of full flavor symmetry of the DPDFs. Let us note that this hypothesis can not be tested in standard QCD fits of diffractive PDFs or in any of the diffractive processes examined with HERA data [23, 24]. Thus, if the measurements at the LHC show the results different from our conclusions, eqs. (201) or (21), there is an interesting possibility that the Pomeron parton distribution, see eq. (8), are not fully flavor symmetric, e.g.

$$
u_{\mathbb{P}}(\beta)=\bar{u}_{\mathbb{P}}(\beta) \neq d_{\mathbb{P}}(\beta)=\bar{d}_{\mathbb{P}}(\beta) .
$$

In such a case the diffractive ratio takes the following form

$$
R_{d i f f}\left(y, \xi_{2}, \xi_{2}\right)=\frac{M_{W}^{2} V_{u d}^{2}}{M_{Z}^{2}} \frac{u_{\mathbb{P}}\left(\beta_{1}, M_{W}\right) d_{\mathbb{P}}\left(\beta_{2}, M_{W}\right)+d_{\mathbb{P}}\left(\beta_{1}, M_{W}\right) u_{\mathbb{P}}\left(\beta_{2}, M_{W}\right)}{C_{u} u_{\mathbb{P}}\left(\beta_{1}^{\prime}, M_{Z}\right) u_{\mathbb{P}}\left(\beta_{2}^{\prime}, M_{Z}\right)+C_{d} d_{\mathbb{P}}\left(\beta_{1}^{\prime}, M_{Z}\right) d_{\mathbb{P}}\left(\beta_{2}^{\prime}, M_{Z}\right)}
$$

where $\beta_{1,2}=x_{1,2} / \xi_{1,2}$ and $\beta_{1,2}^{\prime}=x_{1,2}^{\prime} / \xi_{1,2}$ are the Pomeron momentum fractions carried by the quarks producing the electroweak bosons. The above expression provides a direct sensitivity of the the $d_{\mathbb{P}} / u_{\mathbb{P}}$ ratio, albeit for the quark distributions taken at two different scales, $\mu=M_{W, Z}$. In case of flavor symmetry, the ratio is expected to be approximately a constant, if not, a non-trivial shape may be obtained.

The measurement of the $W$ to $Z$ cross section ratio is sensitive to the $u, d, s$ quark densities in the Pomeron and especially to their ratios. The H1 and ZEUS experiments measured the structure of the pomeron 23, 24]. The fits always assume $u_{\mathbb{P}}=d_{\mathbb{P}}=s_{\mathbb{P}}$ since data are not sensitive to their difference. The measurement of the $W$ to $Z$ cross section will allow to probe this assumption.

In Fig [5 we display the $W$ to $Z$ cross section ratio as a function of $s_{\mathbb{P}} / u_{\mathbb{P}}$ and $d_{\mathbb{P}} / u_{\mathbb{P}}$ ratios while keeping the sum $u_{\mathbb{P}}+d_{\mathbb{P}}+s_{\mathbb{P}}$ constant. We note the strong dependence of the $W$ to $Z$ cross section ratio on the quark density ratio, which will allow to probe the assumption $u_{\mathbb{P}}=d_{\mathbb{P}}=s_{\mathbb{P}}$ using LHC data. In order to show more precisely this dependence, we show in Fig. 6 one projection along a vertical axe: we display the cross section ratio varying for instance $d_{\mathbb{P}} / u_{\mathbb{P}}$ assuming $d_{\mathbb{P}}=s_{\mathbb{P}}$ and $u_{\mathbb{P}}+d_{\mathbb{P}}+s_{\mathbb{P}}$ constant as usual (since this is well constrained by the QCD fits performed at HERA). We notice that the effect of the cross section ratio can be more than a factor of four while varying the quark densities, which shows the potential of such a measurement at the LHC. 


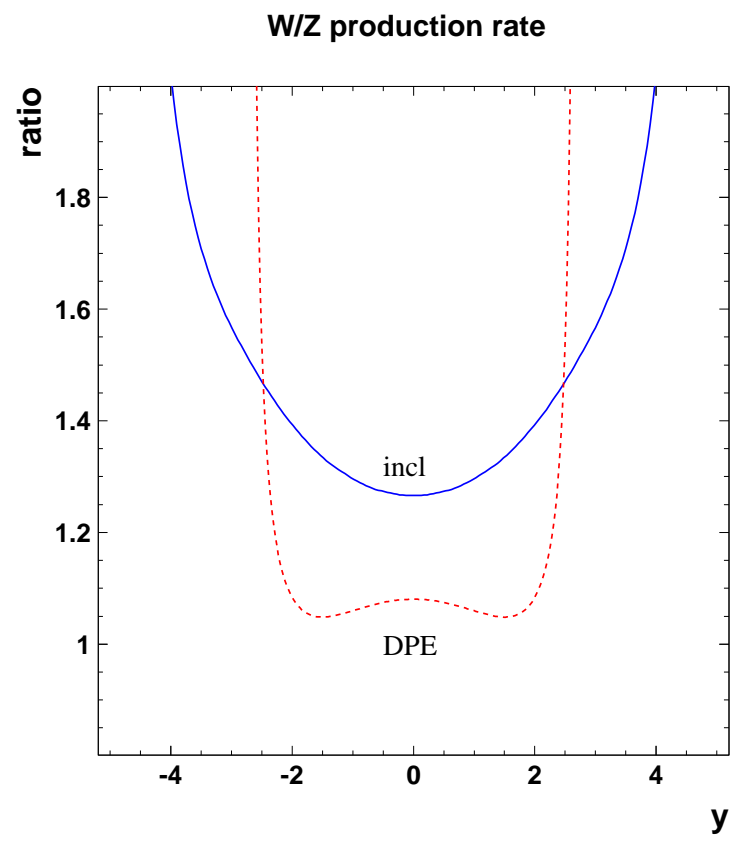

FIG. 4: The $W$ to $Z$ production ratio in the DPE and SCI models (see text).



FIG. 5: The effect of varying the $d / u$ and $s / u$ quark density ratio in the Pomeron on the $W / Z$ cross section ratio keeping $u+d+s$ constant.

\section{CONCLUSION}

We have shown that the double diffractive electroweak vector boson production in the $p p$ collisions at the LHC is an ideal probe of QCD based mechanisms of diffraction. Assuming the resolved Pomeron model, the $W$ production asymmetry in rapidity have been shown to be exactly zero for all rapidities. In other approaches, like the soft color interaction model, in which soft gluon exchanges are responsible for diffraction, the asymmetry is non-zero and equal to that in the inclusive $W$ production. In the same way, the ratio of the $W$ to $Z$ boson production is independent of rapidity in the models with resolved Pomeron and flavor symmetry in contrast to the predictions of the soft color interaction model. The sensitivity to the ratio of the $d$ to $u$ quarks in the Pomeron, using the $W$ to $Z$ cross sections 




FIG. 6: The effect of varying the $d / u$ quark density ratio in the Pomeron on the $W / Z$ cross section ratio keeping $u+d+s$ constant and assuming $d=s$.

ratio, has been studied. Large variations have been found.

\section{Acknowledgments}

This work was supported the grant of Polish National Science Center no. DEC-2011/01/B/ST2/03915.

[1] CDF, F. Abe et al., Phys. Rev. Lett. 74, 850 (1995), hep-ex/9501008].

[2] D0, V. M. Abazov et al., Phys. Rev. Lett. 101, 211801 (2008), [0807.3367].

[3] CDF, T. Aaltonen et al., Phys. Rev. Lett. 102, 181801 (2009), [0901.2169].

[4] G. Aad et al. [ATLAS Collaboration], Phys. Lett. B 701 (2011) 31 arXiv:1103.2929 [hep-ex]].

[5] S. Chatrchyan et al. [ CMS Collaboration ], JHEP 1104 (2011) 050. arXiv:1103.3470 [hep-ex]].

[6] S. Catani, L. Cieri, G. Ferrera, D. de Florian, M. Grazzini, Phys. Rev. Lett. 103 (2009) 082001. arXiv:0903.2120 [hep-ph]].

[7] K. Golec-Biernat, A. Luszczak, Phys. Rev. D81 (2010) 014009. arXiv:0911.2789 [hep-ph]].

[8] M. Boonekamp, F. Chevallier, C. Royon, L. Schoeffel. Feb 2009. 92 pp. Published in Acta Phys.Polon. B40 (2009) 2239-2321

[9] Alexander Kupco, Christophe Royon, Robert B. Peschanski, Phys.Lett. B 606 (2005) 139.

[10] J. C. Collins, Phys. Rev. D 57 (1998) 3051 [Erratum-ibid. D 61 (2000) 019902]; G. Ingelman and P. E. Schlein, Phys. Lett. B 152 (1985) 256.

[11] A. Edin, G. Ingelman, J. Rathsman, Phys. Lett. B366 (1996) 371.

[12] R. K. Ellis, W. J. Stirling and W. B. R., QCD and Collider Physics, Cambridge University Press (1996).

[13] R. S. Thorne, A. D. Martin, W. J. Stirling and G. Watt, arXiv:0907.2387 [hep-ph].

[14] A. Berera and D. E. Soper, Phys. Rev. D50, 4328 (1994), hep-ph/9403276.

[15] A. Berera and D. E. Soper, Phys. Rev. D53, 6162 (1996), hep-ph/9509239.

[16] L. Trentadue and G. Veneziano, Phys. Lett. B323, 201 (1994).

[17] J. C. Collins, J. Huston, J. Pumplin, H. Weerts and J. J. Whitmore, Phys. Rev. D51, 3182 (1995), hep-ph/9406255.

[18] J. D. Bjorken, Phys. Rev. D47, 101 (1993).

[19] M. Boonekamp, V. Juránek, O. Kepka, C. Royon, Proceedings of the Workshop of the Implications of HERA for LHC physics, DESY-PROC-2009-02; arXiv:0903.3861 M. Boonekamp, A. Dechambre, V. Juranek, O. Kepka, M. Rangel, C. Royon, R. Staszewski, arXiv:1102.2531.

[20] M. G. Albrow et al., JINST 4 (2009) T10001; C. Royon, Proceedings of the DIS 2007 workshop, Munich, preprint arXiv:0706.1796. 
[21] V.A. Khoze, A.D. Martin, M.G. Ryskin, Eur. Phys. J. C19 (2 001) 477; Eur. Phys. J. C23 (2002) 311; Eur. Phys. J. C24 (2002) 581; M. Boonekamp, R. Peschanski, C. Royon, Phys. Rev. Lett. 87 (2001) 251806; Nucl. Phys. B669 (2003) 277; A. Dechambre, O. Kepka, C. Royon, R. Staszewski, Phys. Rev. D 83054013 (2011).

[22] O. Kepka and C. Royon, forward Phys. Rev. D 78 (2008) 073005 arXiv:0808.0322 [hep-ph]]. E. Chapon, O. Kepka, C. Royon, Phys. Rev. D81 (2010) 074003; J. de. Favereau et al., preprint arXiv:0908.2020

[23] A. Aktas et al. [H1 Collaboration], Eur. Phys. J. C 48 (2006) 715; S. Chekanov [ZEUS Collaboration], Nucl. Phys. B 800 (2008) 1.

[24] C. Royon, L. Schoeffel, S. Sapeta, R. B. Peschanski and E. Sauvan, Nucl. Phys. B 781 (2007) 1. 